\title{
Desempenho neuropsicológico de tipos de transtorno do déficit de atenção e hiperatividade (TDAH) em tarefas de atenção visual
}

\author{
Neuropsychological performance of attention-deficit hyperactivity \\ disorder (ADHD) subtypes in visual attention tasks \\ Gabriel Coutinho', Paulo Mattos², Catia Araújo $0^{3}$
}

\section{RESUMO}

Objetivo: Comparar o desempenho dos tipos de TDAH em tarefas de seletividade, sustentação e atenção alternada, considerando tempo médio de reação, número de erros por ação e número de erros por omissão em teste computadorizado de atenção visual (TAVIS-III). Métodos: Cento e duas crianças e adolescentes de duas escolas particulares e uma escola pública da cidade do Rio de Janeiro, com idades entre 6 e 17 anos com diagnóstico de TDAH, segundo DSM-IV, foram submetidas ao TAVIS-III. A separação por grupos respeitou os tipos determinados por entrevista semi-estruturada (P-CHIPS), e os resultados foram comparados levando-se em consideração o fator grupo. Resultados: 0 tipo combinado (C) revelou-se o mais comum ( $n=65 ; 63,7 \%$ ), seguido pelo predominantemente desatento (D) ( $n=32$; 31,4\%). O tipo predominantemente hiperativo-impulsivo (HI) foi excluído das análises esta-

\section{Palavras-chaves}

Transtorno do déficit de atenção/hiperatividade, atenção, tipos, testes neuropsicológicos.

\section{Key-words}

Attention deficit/ hyperactivity disorder, attention, subtypes, neuropsychological tests.

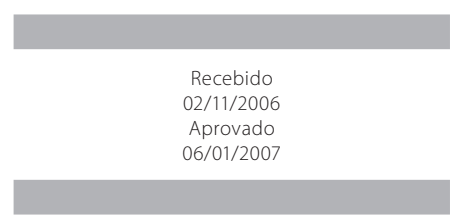
tísticas em virtude da baixa freqüência. O desempenho do grupo C revelou-se inferior apenas em tarefa de atenção sustentada, no que tange ao número de erros por ação e tempo médio de reação ( $p<0,05)$, ambos maiores quando comparados ao grupo D. Conclusão: Apenas em tarefa de atenção visual por tempo mais prolongado, o tipo combinado revelou diferenças significativas em relação ao grupo desatento.

\section{ABSTRACT}

Objective: To compare the effect of ADHD subtypes in tasks of focused, shifted and sustained attention in a visual attention test (TAVIS-III). Indexes of hit reaction time, omission errors and commission errors were considered for each task. Methods: One hundred two children and adolescents aged from 6 to 17 years old recruited from one public and two private schools in the city of Rio de Janeiro with ADHD diagnosis, according to DSM-IV criteria, were evaluated. Three groups were created following the subtypes determined by the semi-structured interview (P-CHIPS). Group performances were compared in order to determine subtype effects. Results: The combined subtype (C) was the most common group $(n=65 ; 63.7 \%)$, followed by the inattentive subtype (I) $(n=32 ; 31.4 \%)$. Hyperactive-impulsive (HI) group was excluded from statistical analysis due to its low frequency. The performance of the C group was found comparatively worse only in the sustained attention task, where subjects presented slower hit reaction time and committed more commission errors $(p<0.05)$ than the I group. Conclusion: Only in longer tasks of visual attention, combined patients had a worse performance than Inattentive ones.

1 Psicólogo, mestrando em Psiquiatria e Saúde Mental pela Universidade Federal do Rio de Janeiro (UFRJ). 2 Psiquiatra, professor adjunto da UFRJ e coordenador do Grupo de Estudos do Déficit de Atenção (Geda) do Instituto de Psiquiatria da UFRJ. 3 Psicóloga e mestre em Informática pela UFRJ. 


\section{INTRODUÇÃO}

Os critérios propostos pelo DSM-IV (American Psychiatric Association, 1994) para o diagnóstico do transtorno de déficit de atenção/hiperatividade (TDAH) contemplam duas dimensões sintomáticas distintas, permitindo os seguintes tipos diagnósticos: com predomínio de desatenção, combinado e com predomínio de hiperatividade-impulsividade. Estudos mostraram algumas diferenças quanto ao impacto das diferentes dimensões sintomáticas, sugerindo que os sintomas de desatenção tendem a se associar a comprometimentos acadêmico (Gadow et al., 2004) e neuropsicológico (Chhabildas et al., 2001; Gadow et al., 2004; Schmitz et al., 2002), ao passo que os sintomas de hiperatividade/impulsividade estão mais associados a problemas comportamentais (Gadow et al., 2004).

Em recente estudo longitudinal com duração de 8 anos, Lahey et al. (2005) demonstraram que o número de sintomas de desatenção tendia a aumentar conforme crianças portadoras de TDAH avançavam nas séries pré-escolares, o que provavelmente estaria associado ao aumento das demandas acadêmicas. Por outro lado, os sintomas de hiperatividade tendiam a diminuir com o passar dos anos. Desta forma, observou-se significativa diminuição do número de indivíduos que atendiam aos critérios para TDAH com predomínio de hiperatividade-impulsividade com o passar dos anos, além de aumento das taxas daqueles com predomínio de desatenção ao final dos 8 anos. Esses achados corroboram os achados de outros estudos, que observaram que os indivíduos com predomínio de desatenção tendiam a ser mais veIhos que aqueles com predomínio de hiperatividade-impulsividade (Chhabildas et al., 2001; Gadow et al., 2004). Gadow et al. (2004) demonstraram ainda que a classificação por tipos pode variar de acordo com o informante, sugerindo que o relato dos responsáveis tende a ser mais preciso que dos professores, uma vez que estes últimos comumente observam os indivíduos apenas em ambiente escolar.

Estudos sugerem que os tipos combinado e com predomínio de desatenção tendem a apresentar déficits neuropsicológicos semelhantes (Chhabildas et al., 2001; Schmitz et al., 2002; Gadow et al., 2004; Nigg et al., 2002), enquanto aqueles com predomínio de hiperatividade-impulsividade não costumam apresentar déficits significativos se comparados a controles normais (Chhabildas et al., 2001; Schmitz et al., 2002; Gadow et al., 2004). Outros autores sugeriram que a divisão por tipos poderia ser feita de acordo com a presença ou ausência de comprometimento das funções executivas em testes neuropsicológicos, uma vez que os portadores que apresentavam tais déficits tendiam a ter comprometimentos mais graves e pior desfecho (Nigg et al., 2005).

Barkley (1997) sugeriu que a dificuldade de inibir comportamentos é uma das principais causas dos déficits aten- cionais e executivos, definindo o TDAH como uma disfunção frontal. Em recente estudo de meta-análise, Willcutt et al. (2005) concluíram que embora déficits em funções executivas estivessem freqüentemente associados ao TDAH, não eram suficientes nem necessários para predizer a presença do transtorno. De fato, algumas amostras de portadores de TDAH não apresentaram déficits significativos em funções executivas quando comparados a controles normais (Gomes et al., 2005; Amaral e Guerreiro, 2001).

O objetivo deste estudo foi o de investigar a influência do tipo no desempenho de portadores de TDAH em um teste de atenção visual (TAVIS-III) (Duchesne e Mattos, 1997), a partir de uma amostra não-clínica. Serão avaliados os seguintes aspectos da atenção visual: seletividade, sustentação e alternância de conceitos. Para cada um dos aspectos, serão investigados índices referentes ao tempo médio de reação, número de erros por ação e número de erros por omissão. O TAVIS-III revelou-se eficiente para discriminar portadores de TDAH de indivíduos normais em estudo anterior com essa mesma amostra não-clínica (Coutinho et al., submetido à publicação).

\section{MÉTODOS}

Realizou-se o estudo com alunos de duas escolas particulares e uma escola pública, todas localizadas na cidade do Rio de Janeiro. As idades variavam entre 6 e 17 anos. Após palestras informativas, os professores encaminharam alunos que apresentavam quaisquer queixa de desatenção e/ou hiperatividade para entrevista semi-estruturada com os pais, visando a investigar a presença de TDAH e de outros transtornos psiquiátricos de eixo I do DSM-IV (P-CHIPS) (Fristad et al., 1998). O Comitê de Ética em Pesquisa do Instituto de Psiquiatria da Universidade Federal do Rio de Janeiro aprovou o protocolo do estudo.

As entrevistas foram coordenadas pelo serviço de orientação educacional (SOE) e foram incluídas as crianças que obtiveram autorização dos pais, de modo seqüencial. As escolas possuíam arquivos acerca da saúde dos alunos, bem como o registro de informações sociofamiliares, incluindo uso de medicamentos ou substâncias psicoativas, que eram verificados com os pais. Crianças e adolescentes com histórico de epilepsia, traumatismo cranioencefálico (TCE), uso de álcool e outras substâncias psicoativas e que estavam utilizando medicamentos de uso neurológico ou psiquiátrico foram excluídas do estudo.

Da amostra inicial de 780 alunos, 102 atenderam aos critérios para TDAH por meio da entrevista com pais e foram posteriormente submetidos ao teste de atenção visual (TAVIS-III), compreendendo as seguintes tarefas:

- Tarefa 1: avaliar a capacidade de focalizar estímulos relevantes, na presença de estímulos distratores, e selecionar a informação para processamento consciente (seletividade). 
- Tarefa 2: avaliar a capacidade de mudar o foco de atenção entre tarefas com demandas cognitivas diferentes, determinando, assim, a qual informação se prestará atenção num dado momento (alternância de conceitos).

- Tarefa 3: avaliar a capacidade de manter a atenção ao longo do tempo. Seria a capacidade de sustentar uma resposta comportamental consistente durante uma atividade contínua (sustentação da atenção).

Tempo médio de reação, número de erros por omissão e número de erros por ação foram computados em cada uma dessas tarefas.

Os portadores foram posteriormente divididos em três grupos de acordo com o tipo determinado pela entrevista com os pais: com predomínio de desatenção, combinado e com predomínio de hiperatividade-impulsividade. Tendo em vista a baixa freqüência de indivíduos com o tipo com predomínio de hiperatividade-impulsividade $(n=5)$, estes foram excluídos das análises estatísticas. Utilizaram-se o Teste $U$ de Mann-Whitney e valor $p$ de 0,05.

\section{RESULTADOS}

A prevalência de TDAH foi maior no sexo masculino $(70,6 \%)$. O tipo combinado foi o mais comum $(63,7 \%)$, seguido do tipo com predomínio de desatenção $(31,4 \%)$. Apenas 5 $(4,9 \%)$ indivíduos atenderam aos critérios para o tipo com predomínio de hiperatividade-impulsividade. Os desatentos eram um pouco mais velhos que os demais tipos, a exemplo do que ocorreu em outros estudos (Chhabildas et al., 2001; Gadow et al., 2004) (Tabela 1).

Seletividade da atenção: não foram observadas diferenças significativas em nenhum dos índices avaliados (tempo médio de reação, número de erros por omissão e número de erros por ação).

Alternância de conceitos: a exemplo do que ocorreu na tarefa de seletividade, nenhum dos três índices apresentou diferença significativa entre os grupos.

Sustentação da atenção: indivíduos do tipo combinado foram significativamente mais lentos e apresentaram um número de erros por ação significativamente maior que os desatentos. Não foram observadas diferenças significativas quanto ao número de erros por omissão. Os resultados estão descritos na tabela 2.

\section{DISCUSSÃO}

A prevalência de TDAH foi maior no sexo masculino que no sexo feminino, corroborando achados de outros estudos (Gomes et al., 2005; Poeta e Neto, 2004; Vasconcelos et al., 2003; Biederman et al., 2005; Chhabildas et al., 2001). A dis-
Tabela 1. Características dos grupos combinado e com predomínio de desatenção

\begin{tabular}{lcc}
\hline & Combinado & Desatento \\
\hline Sexo & $\mathrm{F}=21$ & $\mathrm{~F}=8$ \\
$\mathrm{M}=44$ & $\mathrm{M}=24$ \\
Idade & Média $9,68 \pm 2,02$ & Média $11,34 \pm 3,09$ \\
Escolaridade & Média $3,86 \pm 2,08$ & Média $5,09 \pm 3,00$ \\
\hline
\end{tabular}

F: sexo feminino; M: sexo masculino; escolaridade: anos de escolaridade.

Tabela 2. Médias dos escores em cada índice das tarefas de seletividade, alternância de conceitos e sustentação da atenção visual para os grupos combinado e com predomínio de desatenção

\begin{tabular}{ccccc}
\hline & & Combinado & Desatento & Significância \\
\hline \multirow{2}{*}{ Tarefa 1 } & TMR & 0,57 & 0,56 & .310 \\
& EA & 4,44 & 2,56 & .224 \\
& E0 & 2,50 & 3,38 & .077 \\
Tarefa 2 & TMR & 0,64 & 0,60 & .177 \\
& EA & 6,22 & 6,12 & .144 \\
& EO & 3,33 & 3,08 & .786 \\
Tarefa 3 & TMR & 0,61 & 0,50 & .006 \\
& EA & 8,97 & 4,59 & .000 \\
& EO & 0,27 & 0,09 & .457 \\
\hline
\end{tabular}

A significância foi calculada utilizando 0 Teste $U$ de Mann-Whitney e valor $p$ de 0,05 . Tarefa 1: avalia a seletividade da atenção visual; Tarefa 2: avalia a alternância de conceitos da atenção visual; Tarefa 3: avalia a sustentação da atenção visual; TMR: tempo médio de reação; EA: erros por ação; E0: erros por omissão.

tribuição por tipos revelou maior prevalência do tipo combinado, contrapondo-se a alguns estudos (Chhabildas et al., 2001; Gomes et al., 2005; Poeta e Neto, 2004; Vasconcelos et al., 2003), porém encontrando respaldo em outros estudos também com amostras não-clínicas (Rohde et al., 1999; Biederman et al., 2005).

Não houve influência de gênero no desempenho. Outros estudos, entretanto, já haviam sugerido que em amostras nãoclínicas não há influência de gênero no tipo, idade de início, comorbidades e gravidade do TDAH (Biederman et al., 2005).

Observaram-se poucas diferenças quanto ao desempenho dos tipos combinado e com predomínio de desatenção nas tarefas do TAVIS-III. Outros estudos (Chhabildas et al., 2001; Schmitz et al., 2002; Gadow et al., 2004; Nigg et al., 2002) não demonstraram diferenças significativas entre esses tipos quanto ao desempenho em testes neuropsicológicos, sugerindo que os sintomas de desatenção são responsáveis pelo comprometimento em testes e não os sintomas de hiperatividade-impulsividade. Esses achados parecem estar associados ao comprometimento acadêmico mais grave em indivíduos dos tipos combinado e com predomínio de desatenção se comparados aos hiperativos e controles normais.

As diferenças observadas restringiram-se aos índices de tempo médio de reação e número de erros por ação (impulsividade) em tarefa de sustentação da atenção, com os desatentos apresentando melhor desempenho que os combinados. Nigg et al. (2002) descreveram resultados semelhantes, demonstrando que meninos combinados apre- 
sentavam déficits de controle inibitório, além de lentidão (no índice Stop Signal Reaction Time), quando comparados a meninos do tipo desatento. Esse mesmo padrão não foi verificado quando os tipos foram comparados sem considerar o fator gênero e quando meninas foram comparadas separadamente. Apesar de discretos, tais resultados podem estar associados aos sintomas de hiperatividade/impulsividade, conforme proposto anteriormente (Barkley, 1997).

Em estudo com meninos com idades entre 8 e 10 anos utilizando o Continuous Performance Task (CPT), Collings (2003) verificou que, se comparado ao desempenho dos desatentos e controles sem TDAH, o desempenho dos combinados declinava com o passar do tempo em tarefas mais longas (sustentação da atenção), o que ocasionava mais erros por omissão. Embora os erros observados neste estudo não tenham sido por impulsividade, os achados foram considerados como conseqüência das dificuldades em inibir respostas, que dificultariam a permanência dos combinados nas tarefas mais longas. Os achados podem ser considerados compatíveis com os achados do presente estudo, tendo em vista que as diferenças observadas se restringiram aos índices referentes à tarefa de sustentação da atenção (mais longa).

O estudo foi realizado com amostra não-clínica, o que pode associar-se ao viés de comprometimento mais leve se comparado a amostras auto-referidas. Entretanto, tendo sido utilizados os critérios do DSM-IV para o diagnóstico, não houve inclusão de indivíduos sem comprometimento algum. Deve-se ponderar a ausência de investigação quanto à presença de comorbidades, uma vez que estas são freqüentemente observadas em crianças e adolescentes com diagnóstico de TDAH em nosso meio (Souza et al., 2001). Não se investigou a presença de transtornos de aprendizado, o que deve ser ponderado uma vez que estes podem ocasionar desempenhos deficitários em testes de atenção e funções executivas (Homack e Riccio, 2004; Romine et al., 2004). Outra limitação se deve à ausência de indivíduos do tipo com predomínio de hiperatividade-impulsividade, tendo em vista que a inclusão destes permitiria uma investigação mais acurada da hipótese de que sintomas de desatenção são responsáveis por déficits neuropsicológicos.

\section{CONCLUSÃO}

Os resultados obtidos com o teste TAVIS-III, numa amostra de portadores de TDAH segundo o DSM-IV, sugerem que o desempenho neuropsicológico do tipo combinado é inferior ao do tipo com predomínio de desatenção apenas quando se emprega tarefa mais longa, exigindo níveis maiores de sustentação, à semelhança do que fora anteriormente demonstrado com o teste CPT.

\section{REFERÊNCIAS}

Amaral AH, Guerreiro MM. Transtorno de déficit de atenção e hiperatividade: proposta de avaliação neuropsicológica para diagnóstico. Arq Neuropsiquiatr, 59 (4): 884-8, 2001.

American Psychiatric Association. Diagnostic and Statistical Manual of Mental Disorders: DSM-IV. 4th ed. Washington, DC: American Psychiatric Association; 1994.

Barkley, RA. Behavioral inhibition, sustained attention, and executive functions: constructing a unifying theory of ADHD. Psychological Bulletin, 121 (1): 65-94, 1997.

Biederman J, Kwon A, Aleardi M, Chouinard V, Marino T, Cole H, Mick E, Faraone SV. Absence of gender effects on attention deficit hyperactivity disorder: findings in nonreferred subjects. Am J Psychiatry, 162: 1083-9, 2005.

Chhabildas N, Pennington BF, Willcutt EG. A comparison of the neuropsychological profiles of the DSM-IV subtypes of ADHD. Journal of Abnormal Child Psychology, 29 (6): 529-40, 2001.

Collings RD. Differences between ADHD inattentive and combined types on the CPT. Journal of Psychopathology and Behavioral Assessment, 25(3): 177-89, 2003.

Duchesne M, Mattos P. Normatização de um teste computadorizado de atenção visual. Ara Neuropsiquiatr, 55 (1): 62-9, 1997

Fristad MA, Teare M, Weller EB, Weller RA, Salmon P. Study III: development and concurrent validity of the Children's Interview for Psychiatric Syndromes - parent version (P-ChIPS). J Child Adolesc Psychopharmacol, 8 (4): 221-6, 1998

Gadow KD, Drabick DAG, Loney J, Sprafkin J, Salisbury H, Azizian A, Schwartz J. Comparison of ADHD symptom subtypes as source-specific syndromes. Journal of Child Psychology and Psychiatry, 45 (6): 1135-49, 2004.

Gomes F, Mattos P, Pastura G, Ayrão V, Saboya E. Executive functions in a non-clinical sample of children and adolescents with attention-deficit hyperactivity disorder (ADHD). J Bras Psiquiatr, 54 (3): 178-81, 2005

Homack S, Riccio CA. A meta-analysis of the sensitivity and specificity of the Stroop Color and Word Test with children. Archives of Clinical Neuropsychology, 19: 725-43, 2004

Lahey BB, Pelham WE, Loney J, Lee SS, Willcutt E. Instability of the DSM-IV subtypes of ADHD from preschool through elementary school. Arch Gen Psychiatry, 62: 896-902, 2005.

Nigg JT, Blaskey LG, Huang-Pollock CL, Rappley MD. Neuropsychological executive functions and DSM-IV ADHD subtypes. J Am Acad Child Adolesc Psychiatry, 41 (1): 59-66, 2002.

Nigg JT, Willcutt EG, Doyle AE, Sonuga-Barke EJS. Causal heterogeneity in attention-deficit/ hyperactivity disorder: do we need neuropsychologically impaired subtypes? Biol Psychiatry, 57: 1224-30, 2005.

Poeta LS, Neto FR. Epidemiological study of symptoms of attention deficit/hyperactivity disorder and behavior disorders in public schools of Florianópolis/SC using the EDAH. Rev Bras Psiquiatr, 26 (3): 150-5, 2004.

Rohde LA, Biederman, J, Busnello EA, Zimmermann H, Schmitz M, Martins S, Tramontina S. ADHD in a school sample of Brazilian adolescents: a study of prevalence, comorbid conditions, and impairments. J Am Acad Child Adolesc Psychiatry, 38 (6): 716-22, 1999.

Romine CB, Lee D, Wolfe ME, Homack S, George C, Riccio CA. Wisconsin Card Sorting Test with children: a meta-analytic study of sensitivity and specificity. Archives of Clinical Neuropsychology, 9: 1027-41, 2004

Schmitz M, Cadore L, Paczko M, Kipper L, Chaves M, Rohde LA, Moura C, Knijnik M. Neuropsychological performance in DSM-IV ADHD subtypes: an exploratory study with untreated adolescents. Can J Psychiatry, 47(9): 863-9, 2002.

Souza I, Serra MA, Mattos P, Franco VA. Comorbidade em crianças e adolescentes com transtorno do déficit de atenção. Arq Neuropsiquiatr, 59 (2-b): 401-6, 2001.

Vasconcelos MM, Werner I Jr, Malheiros AFA, Lima DFN, Santos ISSO, Barbosa JB. Prevalência do transtorno de déficit de atenção/hiperatividade numa escola pública primária. Ara Neuropsiquiatr, 61 (1): 67-73, 2003.

Willcutt EG, Doyle AE, Nigg JT, Faraone SV, Pennington BF. Validity of the executive function theory of attention-deficit/hyperactivity disorder: a meta-analytic review. Biol Psychiatry, 57: 1336-46, 2005 